Research Article

\title{
Preparation and Performance Evaluation of Polymeric Microspheres Used for Profile Control of Low-Permeability Reservoirs
}

\author{
Fajun Zhao (D), Hongbao Zhang, Yanping Wu, Dawei Wang, and Yufei Zhang \\ Key Laboratory for Improving Oil and Gas Recovery, Ministry of Education, Northeastern Petroleum University, Daqing, \\ Heilongjiang 163318, China
}

Correspondence should be addressed to Fajun Zhao; fajzhao@126.com

Received 13 July 2019; Accepted 8 February 2020; Published 9 March 2020

Academic Editor: Tingyue Gu

Copyright (c) 2020 Fajun Zhao et al. This is an open access article distributed under the Creative Commons Attribution License, which permits unrestricted use, distribution, and reproduction in any medium, provided the original work is properly cited.

To improve in-depth profile control in a low-permeability reservoir, polymeric microspheres were used. A distillation-precipitation polymerization method was adopted to prepare nanometer-sized polymeric microspheres, whose structure, apparent pattern, thermal endurance, particle size, hydration, and swelling capacity were tested and analyzed by a series of techniques, including infrared spectroscopy, scanning electron microscopy, thermogravimetry, high-pressure and hightemperature rheometry, and dynamic light scattering. The prepared polymeric microspheres were copolymerization products of acrylamide, acrylic acid, and methyl methacrylate that were uniformly round with a centralized size distribution. The nanometersized microspheres had satisfactory hydration/swelling performance, indicating that they could act as oil displacement profile control agents. With the increase of shear rate, the apparent viscosity of the polymeric microspheres was significantly reduced, and the fluid possessed a pseudoplastic behavior. When the shear rate was $100-1000 \mathrm{~s}^{-1}$, the fluid demonstrated a Newtonian fluid behavior. After the polymeric microspheres were hydrated, the particle size distribution curve shows a normal distribution, reaching a maximum swelling size of 21.3 times that of the original microspheres. The plugging performance and deformability of the polymeric microspheres gradually enhanced with swelling time, which makes the microspheres effective pore channel plugging agents for delivering a better in-depth profile control effect in rock cores with lower permeability. The core flooding test showed that, for the heterogeneous core with a permeability of $10 \mu \mathrm{m}^{2}$, polymer microspheres have good plugging effect.

\section{Introduction}

As oil fields are continuously expanding, a heterogeneous reservoir has stronger heterogeneity and offers better distribution of the remaining oil, leading to particularly severe water logging and water channeling. Resultantly, the injected fluid cycle is negatively affected [1-4].

Currently, the commonly used profile control systems fall into several categories: water-soluble polymer gel profile control agent, cross-linked polymer profile control agent, particle profile control agent, precipitation-type profile control agent, foam-type plugging agent, resin-type profile control agent, microbe-type water shut-off and profile control agent, cement-type profile control agent, and secondary cross-linked polymer profile control agent system [5-10]. Routine profile control indicates that it is hard to realize effective plugging. As a new technology for in-depth profile control [11-14], the profile control technology using polymeric microspheres forms elastic microspheres with water swelling properties, owing to polymer cross-linking, and utilizes the microspheres' mechanism of "migrating, plugging, elastic deformation, remigration, and replugging" in porous media to deliver effective plugging control. The microspheres can plug and migrate vigorously in highpermeability zones to deliver the effect of plugging by grade and in-depth profile control. As a newly fashioned profile control technology, in-depth profile control via polymeric microspheres demonstrates excellent profile control and 
displacement performance as well as good formation adaptability; thus, it has become a hot topic for both domestic and international research.

Related laboratory experiments proved that polymeric microspheres can expand, physically adsorb and cross-link, and demonstrate salinity and shear resistance $[15,16]$. In contrast to traditional chemical agents, polymeric microspheres are characterized by small initial particle sizes, slow expansion, salinity tolerance, and shear resistance. Therefore, they are able to enter plug formation depths to change the existing flow direction of injected water and enlarge swept volume, thereby improving water drive development.

To eliminate the technological drawbacks of existing polymeric microspheres used for low-permeability reservoir control and provide microspheres with stronger in-depth profile control capacity, the authors adopted a distillation-precipitation polymerization method to prepare micrometer-sized polymeric microspheres. The resulting particles were studied using infrared analysis, scanning electron microscopy, and dynamic light scattering to study the pattern, particle size distribution, hydration/swelling properties, and rheological properties of the micrometersized polymeric microspheres.

\section{Experiment}

2.1. Materials and Instrumentation. Acrylamide (AM), acrylic acid (AA), methyl methacrylate (MMA), N,Nmethylene bis-dialkyl-phosphonate acrylamide (MBA), azoisobutyronitrile (AIBN), acetonitrile, and ethanol were all obtained in analytical grade from Sinopharm Chemical Reagent Co., Ltd.

Sodium hydroxide and hydrochloric acid were obtained in analytically pure grade from Sinopharm Chemical Reagent Co., Ltd. The mineralization degree of the simulated formation water was $8000 \mathrm{mg} / \mathrm{L}$.

The following instrumentation was used: FEI Quanta 450 SEM with a resolution of $10 \mathrm{~nm}$ and an operating voltage of $20 \mathrm{kV}$ (American FEI Company); Alpha 2-4 vacuum freeze drier (German Martin Christ Company); iS10 FT-IR spectrometer produced by American Nicolet Company, with a scanning range of 4000 to $400 \mathrm{~cm}^{-1}$ and application of $\mathrm{KBr}$ pellet-pressing technology; and Zetasizer Nano ZS 90 dynamic light scattering particle characterizer (British Malvern Company) with a $10 \mathrm{~mW} \mathrm{He}-\mathrm{Ne}$ laser, test wavelength of $633 \mathrm{~nm}$, test scope of $0.02-2000 \mu \mathrm{m}$, and test temperature of $25^{\circ} \mathrm{C}$.

2.2. Preparation of the Polymeric Microspheres. The polymeric microspheres were prepared according to the literature $[17,18]$ : $A M$ and AA served as the monomers, MBA served as the cross-linking agent, AIBN served as the radical initiator, and the distillation-precipitation polymerization reaction occurred in acetonitrile at $90^{\circ} \mathrm{C}$. The state of ebullience was maintained for $15 \mathrm{~min}$ to wait for the reactant liquor to turn light blue and then become milky. The temperature of the oil bath was adjusted to $115^{\circ} \mathrm{C}$, and its reflux ratio was kept at about 2 . The reaction lasted for $2 \mathrm{~h}$, almost to dryness. The white solid powder precipitate was dispersed by ethanol, washed, centrifugated, and then dried in vacuum at room temperature for $12 \mathrm{~h}$ to obtain the polymeric microspheres. After the drying process, the polymer microspheres obtained can be guaranteed to be water-free.

Compared with the preparation method of microspheres obtained by polymerization in microemulsion, the precipitation polymerization method does not need any stabilizer or surfactant, the surface of the product obtained is clean, and the posttreatment process is simple. The polymer microspheres prepared have good water swelling properties; they have elasticity and deformability after water absorption and swelling and are suitable for deep profile oil displacement, strong temperature and salt resistance, and stable structure.

Ternary polymerization of AM, AA, and MMA was carried out using radical copolymerization. The copolymerization reaction equation, as initiated by AIBN, in the presence of cross-linking agent MBA, is as follows.

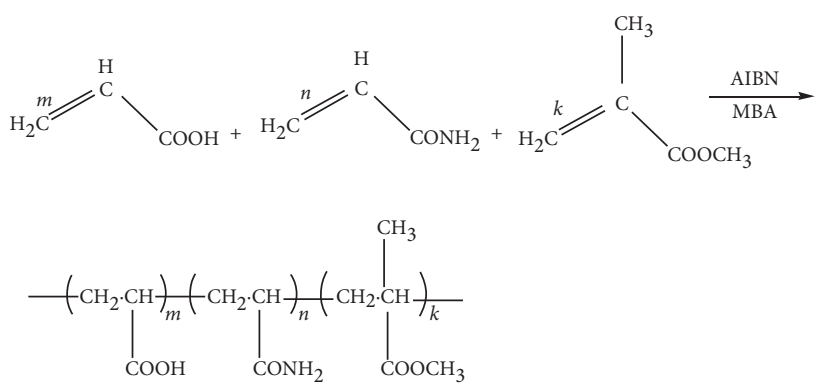

By changing the polymerization conditions, such as initiator, cross-linking agent, monomer concentration, and monomer ratio, AM/AA/MMA polymeric microspheres with sizes ranging from $200 \mathrm{~nm}$ to $2 \mu \mathrm{m}$ could be obtained. The preparation conditions of the polymeric microspheres used in this experiment were as follows: the mass ratio of components AM/AA/MMA was $5: 2: 3$, the mass fraction of the cross-linking agent was $5 \%$, the total mass concentration of the monomer was $25 \mathrm{~g} / \mathrm{L}$, and the mass of the initiator was $0.02 \mathrm{~g}$. The image of polymer microspheres as oil displacement profile control agent is shown in Figure 1.

\subsection{Experimental Method}

2.3.1. FT-IR Analysis. A Nicolet iS10 infrared spectrophotometer was used to determine the infrared absorption spectra. The samples were prepared as potassium bromide pellets, and 20 scans were taken with $21 \mathrm{~cm}^{-1}$ resolution. The composition and structure were analyzed using the Spectrogram program.

\subsubsection{Environmental Scanning Electron Microscope (ESEM)} Image Analysis. The polymeric microsphere solution (approximately $0.1 \mathrm{~mL}$ ) was added dropwise to a concave copper sample table of the Quanta 450 field emission ESEM. 


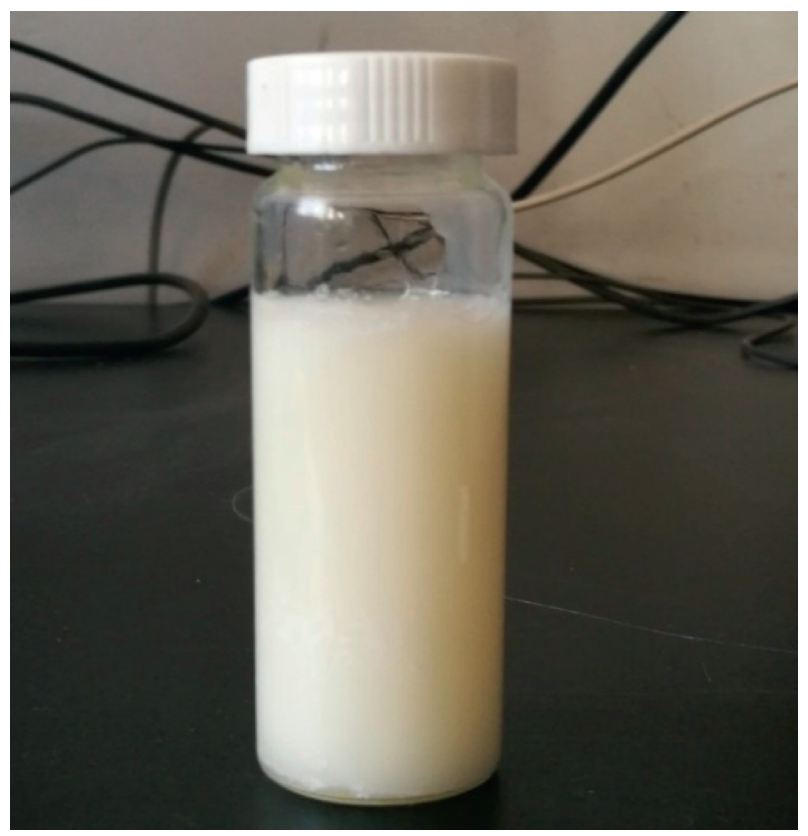

Figure 1: Image of the polymer microsphere as an oil displacement profile control agent.

The samples were sealed to prevent volatilization of the solvent, and the temperature in the sample compartment was rapidly reduced to $-196^{\circ} \mathrm{C}$ using liquid nitrogen. The sample table containing samples was placed in an Alpha 2-4 vacuum freeze drier sample chamber and lyophilized for $24 \mathrm{~h}$. The resulting residue was assumed to accurately model the structure of the in-solution polymer and was analyzed using SEM.

2.3.3. Thermogravimetric Analysis. The thermal stability of the polymer was analyzed by TG209 thermogravimetric analyzer from Netzsch, Germany. The temperature range was $20 \sim 700^{\circ} \mathrm{C}$, the heating rates range from 0.1 to $40 \mathrm{~K} / \mathrm{min}$, which ensures that the data obtained are sufficiently extensive for thermogravimetric analysis of the polymer and during the whole analysis process, and the heating rate remains constant as a set value. The temperature control accuracy was $\pm 0.1^{\circ} \mathrm{C}$, and the thermogravimetric balance reading accuracy was $\pm 0.001 \mathrm{mg}$.

2.3.4. Analysis of Rheological Properties. The rheological properties of polymeric microsphere solutions were tested using a Haake RS-6000 rheometer in conjunction with a $\mathrm{C} 60 / 1^{\circ} \mathrm{Ti}$ neural plate sensing system. The frequency scanning range was set to $0.01-100 \mathrm{HZ}$, and data acquisition was computer-controlled. The relation of shear rate with shearing stress and apparent viscosity was determined.

2.3.5. Size Analysis of the Polymeric Microspheres. The AM/ AA/MMA polymeric microsphere solution with a mass concentration of $1.5 \mathrm{~g} / \mathrm{L}$ was formulated for hydration at $55^{\circ} \mathrm{C}$ for various periods of time. The Zetasizer Nano ZS 90 laser particle size analyzer was preheated for $30 \mathrm{~min}$, and the
AM/AA/MMA microsphere solutions were placed in the sample cell to determine the size distributions of the polymeric microspheres.

2.3.6. Test of Hydration and Swelling Performance. Different volumetric flasks were used to formulate solutions whose concentration of AM/AA/MMA polymeric microspheres was $1.5 \mathrm{~g} / \mathrm{L}$ with $\mathrm{NaCl}$ mass concentrations of 5,10 , 15 , and $20 \mathrm{~g} / \mathrm{L}$. The solutions were placed in an incubating shaker at $60^{\circ} \mathrm{C}$ for hydration and swelling. The AM/AA/ MMA polymeric microsphere solutions were subjected to dynamic light scattering characterization at various time points to monitor the swelling size distributions.

\section{Experimental Results and Discussion}

3.1. Structure Characterization of the Polymeric Microspheres. The infrared spectra of AM/AA/MMA polymeric microspheres are shown in Figure 2. The two broad peaks at 3378 and $3197 \mathrm{~cm}^{-1}$ can be assigned to the $\mathrm{N}-\mathrm{H}$ primary amide vibration. The two peaks at 2954 and $1451 \mathrm{~cm}^{-1}$ can be assigned to the existence of a methyl group $(\mathrm{C}-\mathrm{H}$ stretching). The super strong absorption peaks at 1667 and $1191 \mathrm{~cm}^{-1}$ are characteristic absorption peaks of a carboxylic acid ester $\left(1667 \mathrm{~cm}^{-1}\right.$ can be assigned to the $\mathrm{C}=\mathrm{O}$ stretch). The peak at $1043 \mathrm{~cm}^{-1}$ can be assigned to the $\mathrm{O}-\mathrm{H}$ out-ofplane bending vibration. The above characteristic IR vibration peaks indicate that this substance is the polymer product of AM, AA, and MMA copolymerization.

3.2. Thermal Stability Analysis of Polymer Microspheres. The polymer microspheres were water-free before the thermal stability analysis. The TGA curve of polymeric microspheres is shown in Figure 3. From Figure 3, it can be seen that the thermogravimetric curve of polymeric microspheres can be divided into three stages. In the first stage, the temperature range of 5\% TGA of polymeric spheres is $25-106^{\circ} \mathrm{C}$, which is caused by the volatilization of adsorbed water on the surface of polymeric microspheres. In the second stage, the temperature range is $106-330^{\circ} \mathrm{C}$, and the initial decomposition temperature is $106^{\circ} \mathrm{C}$, which can be attributed to the fracture and decomposition of amido and carboxyl groups in polymer molecular chain of polymeric microspheres. In the third stage, the temperature range is $330-680^{\circ} \mathrm{C}$, and the initial decomposition temperature is $330^{\circ} \mathrm{C}$, which is caused by the fracture and decomposition of the polymer molecular main chain. TGA data analysis shows that polymeric microspheres have good thermal stability.

3.3. SEM Characterization of the Polymer Microsphere Gel. The low-magnification image of the AM/AA/MMA polymeric microspheres is shown in Figure 4. The polymeric microspheres are uniformly round with good sphericity, being microspheres in a real sense. The polymeric microspheres are dozens to hundreds of nanometers in size. 


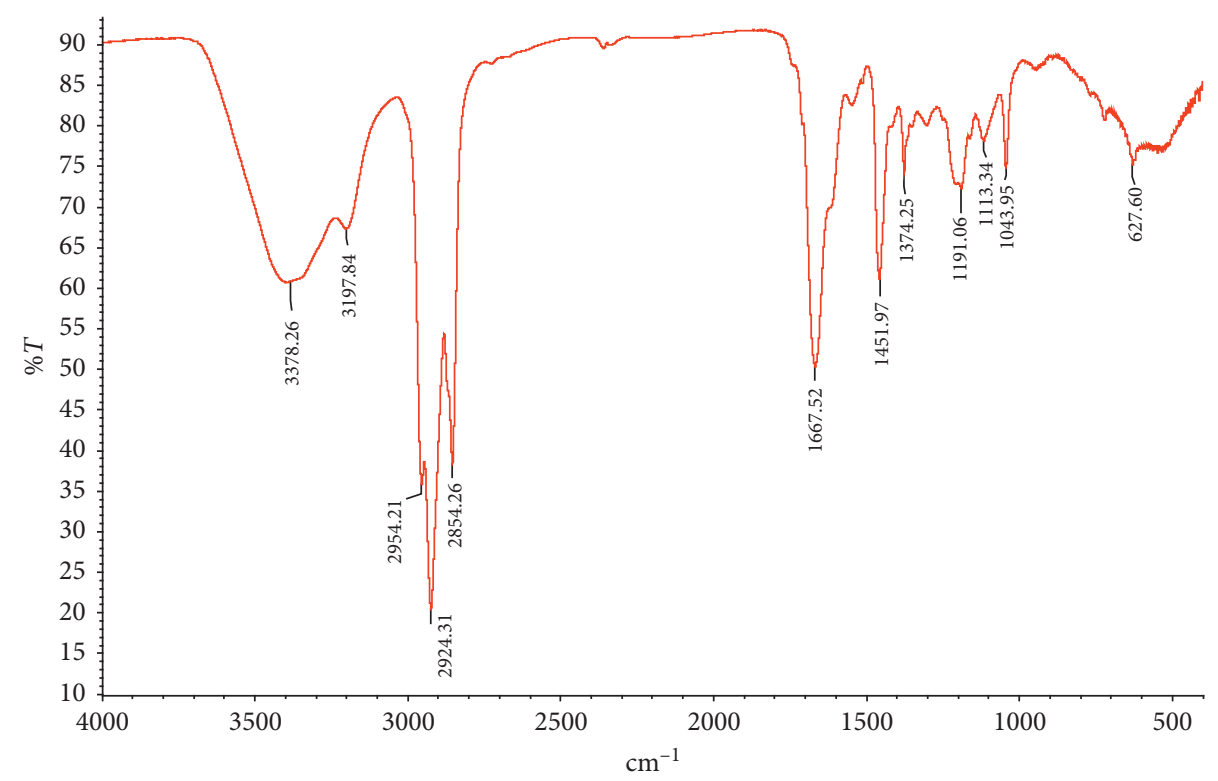

FIGURE 2: Infrared spectra of the polymeric microspheres.

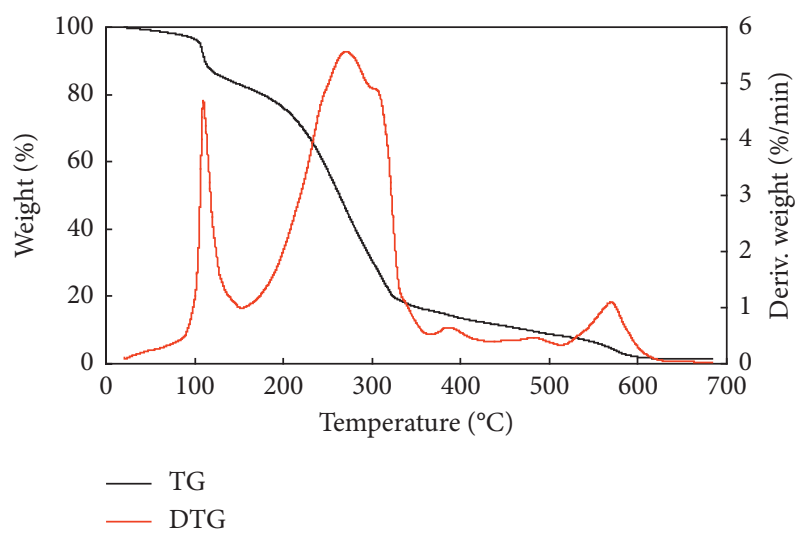

FIgURE 3: TGA-DTG curve of the polymeric microspheres.

3.4. Hydration and Swelling Properties of the Polymeric Microspheres. Polymeric microspheres were evenly dispersed in simulated formation water with a mineralization degree of $8000 \mathrm{mg} / \mathrm{L}$ to achieve a concentration of $0.5 \%$. The formulated solution was placed in an electro-thermostatic blast oven at $60^{\circ} \mathrm{C}$ for hydration and swelling for $1,3,5,10$, 15 , and $20 \mathrm{~d}$. SEM was then used to investigate the characteristics of the polymeric microsphere solutions after various days of hydration and swelling, as shown in Figure 5.

Figure 5 shows that the polymeric microspheres become spatially reticulated from the initial round form after hydration and swelling in $0.5 \%$ simulated formation water. The polymeric microsphere solutions were placed at a constant temperature of $60^{\circ} \mathrm{C}$ for hydration and swelling. As the hydration and swelling time increased, the polymeric microspheres gradually hydrated, and the edges gradually became blurred. At the same time, with the increase of the hydration expansion time, the polymer microspheres exhibit more serious mutual adhesion. Reflected in the SEM photos, the meshes are dense. At this time, the meshes are circular meshes with strong energy, and the self-similarity of the fractal growth is stronger. This is mainly due to the gradual close-up of isolated polymer clusters, and the clusters are closely packed and intersected and overlapped with each other. The limit between microsphere surfaces was already difficult to distinguish under the initial conditions; however, with increasing aging time, the surface limits become even more occluded. As hydration and swelling time increased, the polymeric microspheres began to congeal, resulting in the dense meshes observed in the SEM images. This was primarily attributed to the polymer coils closely packing into overlapping and entangling structures as the microspheres swelled [19]. Forces, such as intermolecular hydrogen bonding, caused the macromolecular chain segments of separate microspheres to entangle, leading to physical crosslinking. Chain bundles that were formed by hydrophobic interactions constituted each reticulation mesh. Nodes existed within the meshes. The chain bundles, meshes, and nodes combined to create a stereoscopic spatial reticulation. After full swelling, the polymeric microspheres possessed a certain microviscosity. Furthermore, their mutual interactions may prove helpful for plugging high-permeability channels and large pore paths in reservoir rock.

3.5. Rheological Properties of the Polymeric Microspheres. The shear stress-shear rate relation curve and apparent viscosity-shear rate relation curve for the polymeric microspheres at $60^{\circ} \mathrm{C}$ are shown in Figure 6.

When the shear rate increased from 0 to $100 \mathrm{~s}^{-1}$, the apparent viscosity of the polymeric microspheres decreased significantly, and the fluid behaved like a pseudoplastic fluid. This can be explained as follows: the dispersed systems formed by the polymeric microspheres all appeared as ordered layer structures, and the particles were mutually independent and flowed in the interlayer space. The applied tangential stress could orient the particles to a certain extent, 


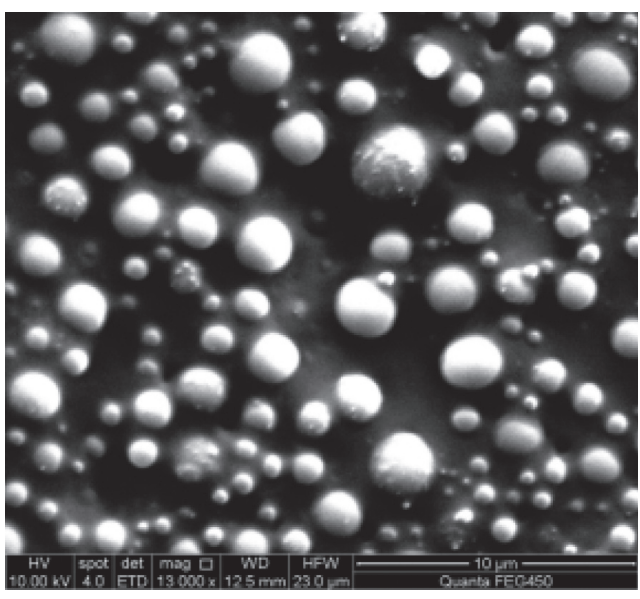

(a)

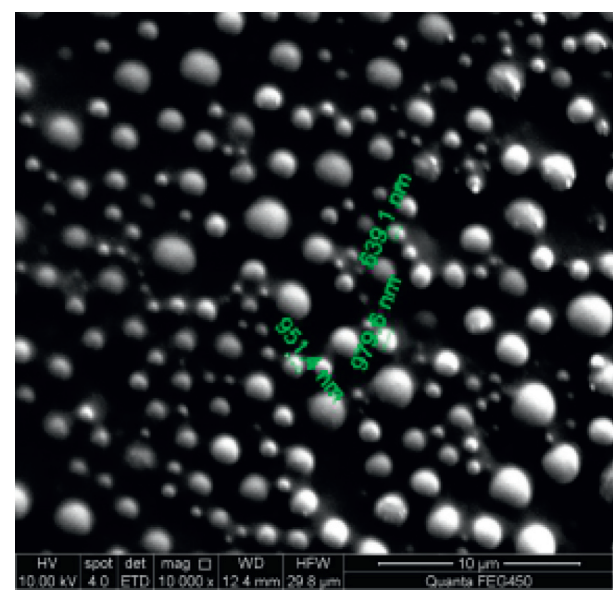

(b)

FIgURE 4: Low-magnification SEM image of the polymeric microspheres.

thereby limiting the deformation degree of the particles to the layers where they were located, so the system demonstrated shear thinning, a characteristic of a pseudoplastic fluid.

When the shear rate increased from 100 to $1000 \mathrm{~s}^{-1}$, the apparent viscosity for the polymeric microspheres decreased slowly, and the apparent viscosity did not change significantly. In this range, the fluid appears to approximate a Newtonian fluid. This can be explained as follows: when the shear rate increases, the ordered laminated structure of the dispersed system formed by the polymeric microspheres was disorganized into a disordered dispersed structure. The cross-linking points in the internal microsphere structure limited the degree of deformation such that the microspheres did not spread out extensively to become linear. The shearing stress was temporarily responsible for microsphere binding, and the slight intermolecular forces from the polymeric microspheres themselves assisted in particulate aggregation. However, the shearing stress would also disconnect particulate aggregation. When the forming function of shearing stress for polymeric microsphere aggregation and the dissociation function reach a dynamic balance, the increase of the shear rate does not significantly change the apparent viscosity of the polymeric microsphere systems, and the fluid appears Newtonian.

3.6. Analysis of Microsphere Size Distribution. The change rule for polymeric microsphere size with hydration/swelling time at a constant temperature of $60^{\circ} \mathrm{C}$ and the swelling ratio of polymeric microspheres were calculated. Understanding these parameters would assist in the matching of polymeric microsphere systems to reservoirs and optimization of the solution injection parameters.

The size distributions of the AM/AA/MMA polymeric microspheres are shown in Figure 7. The average particle size of polymeric microspheres is shown in Table 1. In the initial state of Table 1, the size of the polymeric microspheres was $380.4-387.3 \mathrm{~nm}$, and PDI was 0.622-0.737. After hydration for $1 \mathrm{~d}$, the volumetric distribution curve of the AM/AA/
MMA microspheres could be described as a normal distribution ranging from 2.534 to $2.658 \mu \mathrm{m}$, and PDI was $0.278-0.646$. After hydration for $20 \mathrm{~d}$, the volumetric distribution curve of the AM/AA/MMA microspheres became 8.019-8.256 $\mu \mathrm{m}$, and PDI was $0.281-0.342$, expanding approximately 21.3 times their original size, indicating that the polymeric microspheres had a certain hydration and swelling capacity. From PDI data, it can be seen that, with the increase of hydration time, the dispersion degree of polymeric microspheres becomes more and more uniform. The initial particle sizes of the polymeric microspheres were hundreds of nanometers; thus, injection performance should be excellent, and they should smoothly pass through narrow pore openings to penetrate the depth of the reservoir formations. After hydration and swelling at a constant temperature of $60^{\circ} \mathrm{C}$, the volume of the polymeric microspheres increases significantly, and the average particle size bulks up to the micrometer scale. This should meet the requirement for plugging the pores in the rock formation. The average particle size of polymeric microspheres is shown in Table 1.

\subsection{Experiment on Plugging Performance of Polymer Microspheres}

3.7.1. Experimental Conditions and Methods. The following experimental conditions were used: the experimental temperature was $75^{\circ} \mathrm{C}$, the experimental water was simulated formation water, the mineralization was $8000 \mathrm{mg} / \mathrm{L}$, and the polymer microspheres were self-made samples. The experimental core was a one-dimensional homogeneous artificial core. The core size was $25 \mathrm{~mm} \times 100 \mathrm{~mm}$, and the gas permeability was $10 \times 10^{-3} \mu \mathrm{m}^{2}$.

(1) Experimental Method. The core displacement process was connected, installed, and debugged, the model was drained and the water was saturated, the core porosity was calculated, and then the simulated formation water was injected into the core at a rate of $0.2 \mathrm{~mL} / \mathrm{min}$. The pressure was 


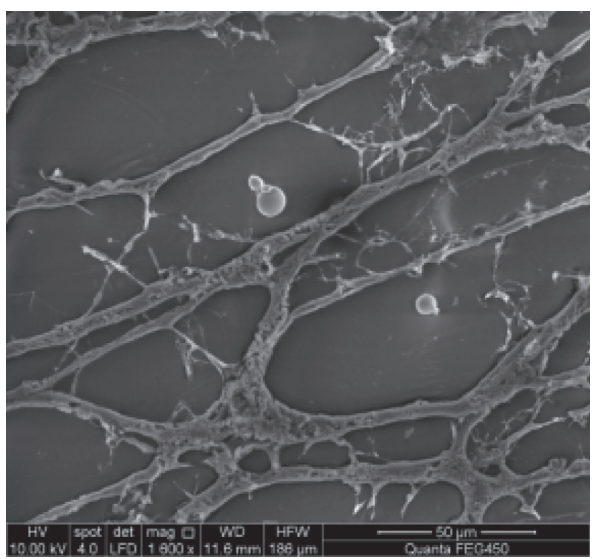

(a)

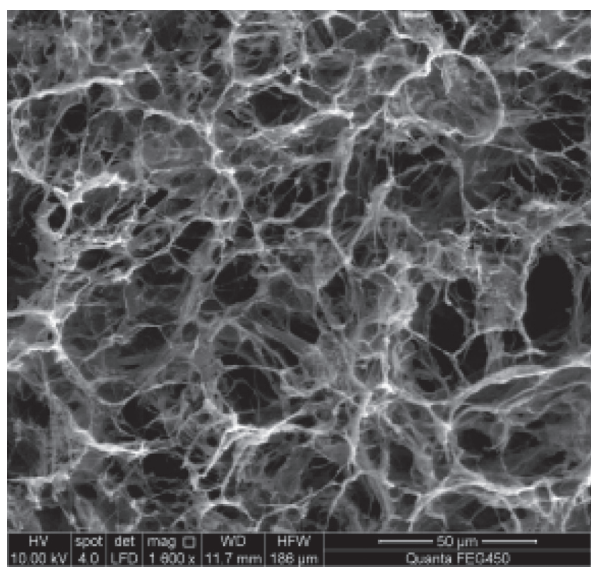

(c)

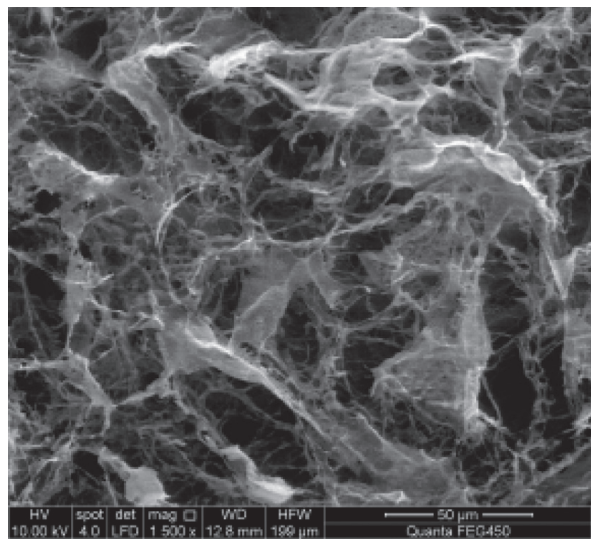

(e)

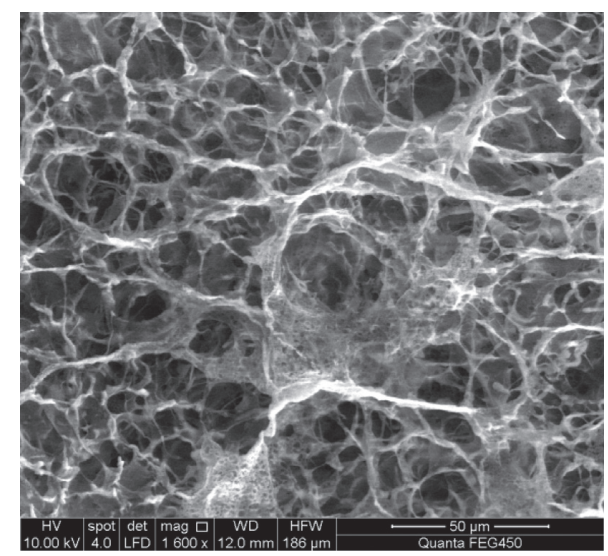

(b)

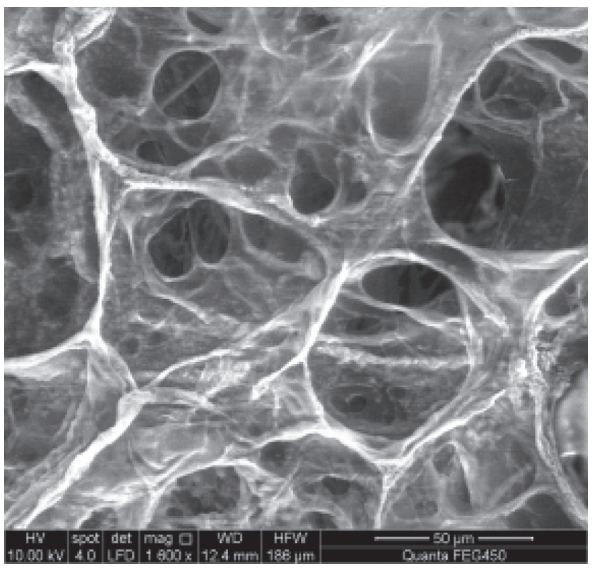

(d)

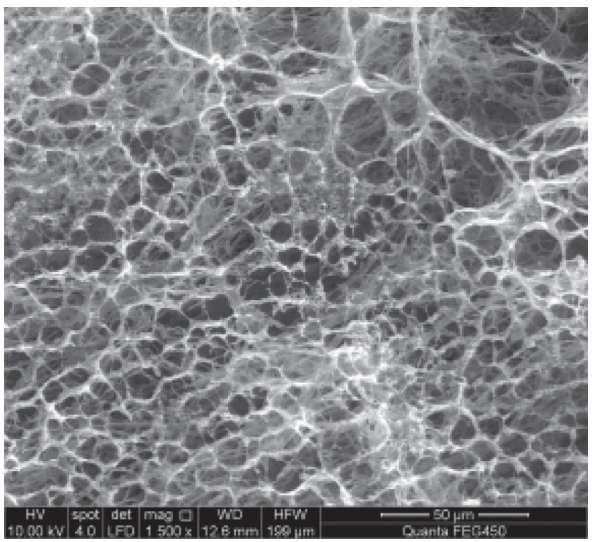

(f)

Figure 5: Polymeric microsphere solution after hydration and swelling in simulated formulation water over time. (a) Polymeric microsphere solution before aging in simulated formation water. (b) Polymeric microsphere solution after aging for $1 \mathrm{~d}$. (c) Polymeric microsphere solution after aging for $5 \mathrm{~d}$. (d) Polymeric microsphere solution after aging for $10 \mathrm{~d}$. (e) Polymeric microsphere solution after aging for $15 \mathrm{~d}$. (f) Polymeric microsphere solution after aging for $20 \mathrm{~d}$.

recorded after the pressure sensor reading is stable, and the polymer microsphere solution was configured with a concentration of $0.2 \%$ and $0.5 \%$; the polymer microsphere solution was injected with a concentration of $0.2 \%$ at the same flow rate, with the total injection volume being $0.3 \mathrm{PV}$, and the pressure gradient was recorded. Water was injected, and the pressure was recorded after the pressure gauge reading is stable; then the polymer microsphere solution was injected with a concentration of $0.5 \%$ at the same flow rate, with the total injection volume being $0.3 \mathrm{PV}$, and the pressure gradient was recorded between the two ends of the core during the injection. Filling was continued. After the pressure gauge reading is stable, the pressure was recorded and the permeability of the core was calculated after the 


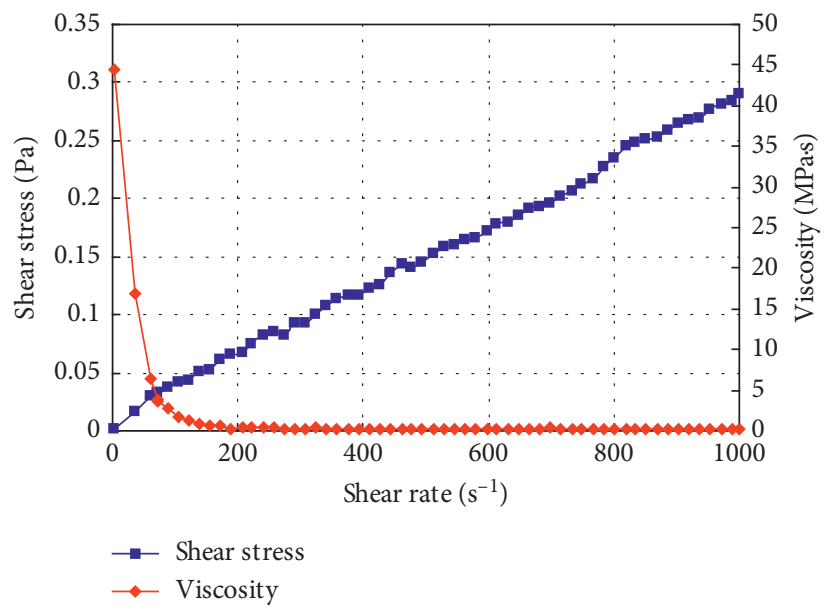

Figure 6: Curves for shear stress and apparent viscosity with shear rate at $60^{\circ} \mathrm{C}$.
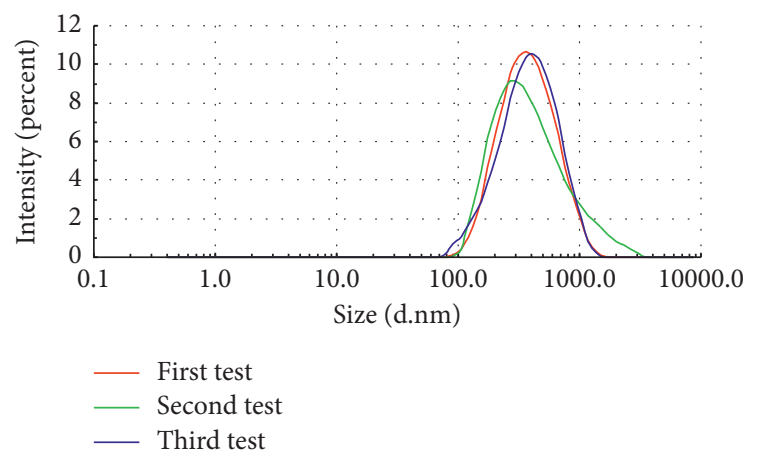

(a)

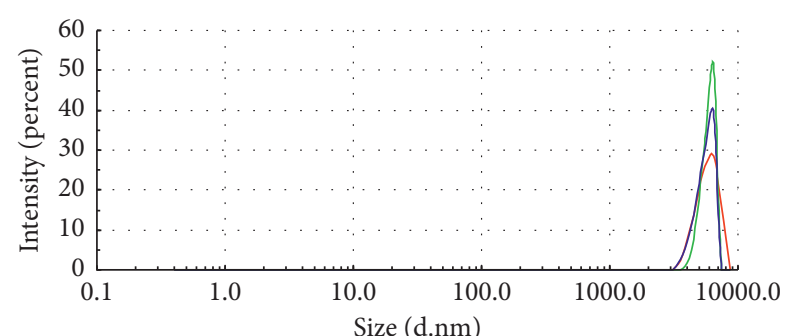

— First test

_ Second test

— Third test

(c)

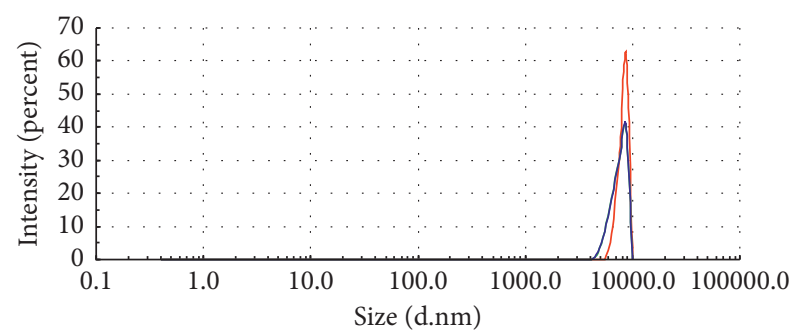

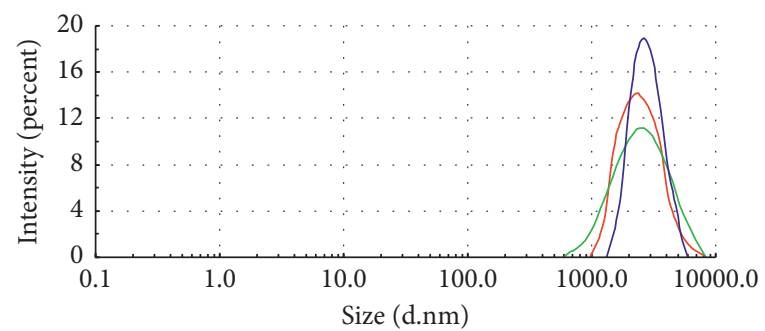

- First test

Second test

_ Third test

(b)

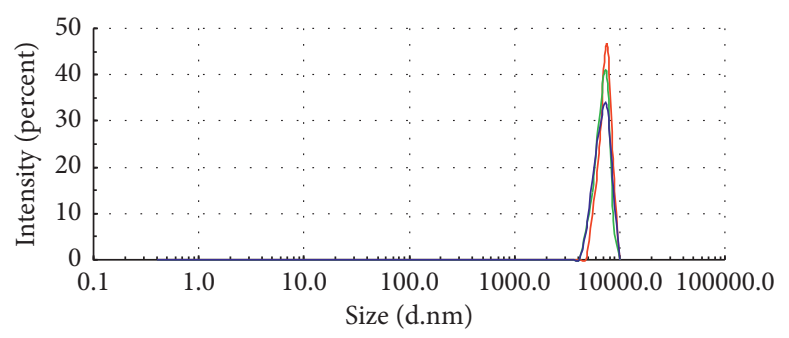

— First test

— Second test

_ Third test

(d)

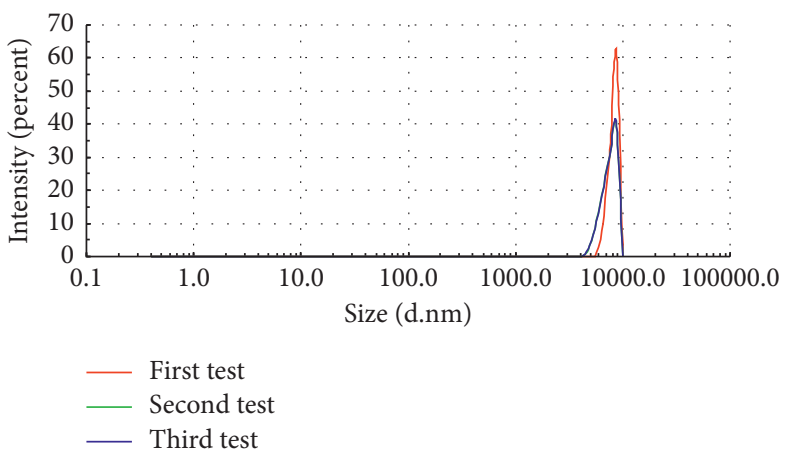

(f)

(e)

FIgURe 7: Size distribution diagram of the polymeric microsphere solutions over time. (a) Original size distribution of the polymeric microsphere solution. (b) Size distribution of the polymeric microsphere solution after aging for $1 \mathrm{~d}$. (c) Size distribution of the polymeric microsphere solution after aging for $5 \mathrm{~d}$. (d) Size distribution of the polymeric microsphere solution after aging for $10 \mathrm{~d}$. (e) Size distribution of the polymeric microsphere solution after aging for $15 \mathrm{~d}$. (f) Size distribution of the polymeric microsphere solution after aging for $20 \mathrm{~d}$. 
TABle 1: Average particle sizes of the polymeric microspheres.

\begin{tabular}{|c|c|c|c|c|c|c|c|c|}
\hline \multirow{2}{*}{ Hydration time (day) } & \multicolumn{4}{|c|}{ Average particle size (nm) } & \multicolumn{4}{|c|}{ PDI (polymer dispersion index) } \\
\hline & First test & Second test & Third test & Average & First time & Second time & Third time & Average \\
\hline Initial & 380.4 & 371.2 & 387.3 & 379.6 & 0.622 & 0.737 & 0.641 & 0.667 \\
\hline $1 \mathrm{~d}$ & 2534 & 2515 & 2658 & 2569 & 0.475 & 0.646 & 0.278 & 0.466 \\
\hline $5 \mathrm{~d}$ & 6460 & 6713 & 6569 & 6581 & 0.287 & 0.206 & 0.218 & 0.237 \\
\hline $10 \mathrm{~d}$ & 7396 & 7302 & 7224 & 7307 & 0.331 & 0.297 & 0.312 & 0.313 \\
\hline $15 \mathrm{~d}$ & 7826 & 7545 & 7569 & 7647 & 0.238 & 0.249 & 0.273 & 0.253 \\
\hline $20 \mathrm{~d}$ & 8256 & 7991 & 8019 & 8089 & 0.281 & 0.34 & 0.342 & 0.321 \\
\hline
\end{tabular}

polymer microspheres are injected. The polymer microspheres with a concentration of $0.2 \%$ and $0.5 \%$ were placed in a constant temperature blast drying oven at a constant temperature of $75^{\circ} \mathrm{C}$; the polymers were taken out on days 1 , $5,10,15$, and 20, respectively. Microsphere solutions were used to test the sealing performance of polymers at different ages.

3.7.2. Results Analysis of Plugging Performance of Polymer Microspheres. Figure 8 shows that, during the initial water flooding, the injection pressure began to increase. After the water-flooding pressure was stabilized, the polymer microspheres with a concentration of $0.2 \%$ were injected, and the injection pressure began to increase. When the pressure increased to a certain level, the microspheres deformed through the throat; then the injection of polymer microspheres with a concentration of $0.5 \%$ showed that the pressure continued to increase. After the pressure rises to a certain value, the microspheres deform again and move forward through the throat. With the continuous injection of polymer microspheres, the number of polymer microspheres in the core continues to increase. Although the polymer microspheres also constantly deform and migrate, the result of the combined action of the polymer microspheres is that the injection pressure continues to increase; in subsequent water flooding, the injection pressure basically maintains the high level when the polymer microspheres are injected.

The injectability of the unaged polymer microsphere solution in the core is very good, which shows that no pipeline blockage occurs during the injection process, the microspheres can smoothly enter the core, the injection pressure gradually increases, and there is no large fluctuation. When polymer microsphere solutions are injected into the core after different aging periods, it can be seen that the injection pressure is significantly increased, indicating that the polymer microspheres can smoothly enter the core and plug. However, after long-term aging, the polymer microsphere solution cannot be injected into the core smoothly. Instead, a large area and a large amount of residue remain on the end face of the core, forming a surface blockage, which causes the pressure to rise continuously (Table 2).

\section{Profile Control Mechanism}

The polymeric microspheres can act as a cross-linked expansive plugging agent with excellent selective pore-entering capacity after imbibition [20]. After swelling, if the

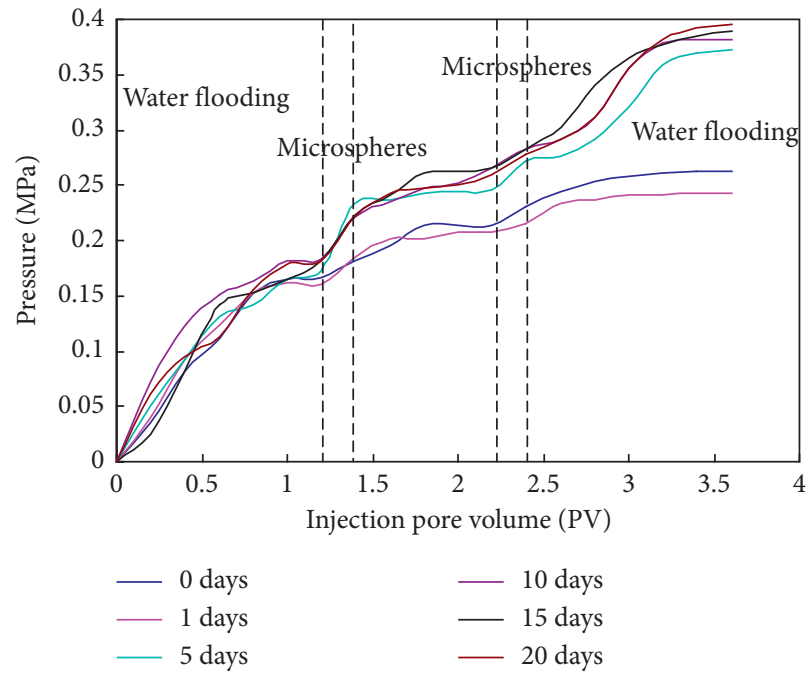

FIGURE 8: Plugging pressure curve of polymer microsphere system.

polymeric microspheres were still smaller than $1 / 3$ of the pore diameter, the polymeric microspheres passed smoothly through. If the microsphere diameter was greater than $1 / 3$ of the pore diameter, they passed through slowly and fractured to a certain extent in cases where the pressure was large. The polymeric microspheres deformed under the driving pressure in the immediate vicinity of the wellbore because of the large pressure difference after the microspheres were injected into an oil layer pore. Once injected, the microspheres entered the depth of the oil layer via pores in the oil layer. As the pressure difference under the oil layer is small, the polymeric microspheres were retained in the pores, thereby plugging the pore channel. This is the mechanism of profile control. Profile control by the polymeric microspheres [21-24] involves a deformation-driven mechanism, a pressure fluctuation mechanism, and a shear fracture mechanism. In fact, this mechanism is often the comprehensive manifestation of several mechanisms when the microspheres pass the pore opening chokepoint, and it is difficult to attribute functioning to a single mechanism. The most visible aspect is the physical plugging of a high-permeability layer; i.e., after water absorption, the polymeric microspheres enter the depth of the rock formation to form unevenly sized swollen bodies. In the high-permeability zone, the large polymeric microspheres can plug the large channel to force the current to turn and spread to mediumand low-permeability zones, thereby expanding the area of displacement in the oil reservoir. In the medium- and low- 
TABLe 2: Advantages and disadvantages of profile control of polymeric microspheres.

At the initial stage of polymeric microspheres injection, the microspheres are very small and can enter the deep formation smoothly with the solution. They can absorb water and expand in the water. They have the characteristics of migration, plugging, elastic deformation, remigration, and replugging in the pore throat of the reservoir, and they have strong
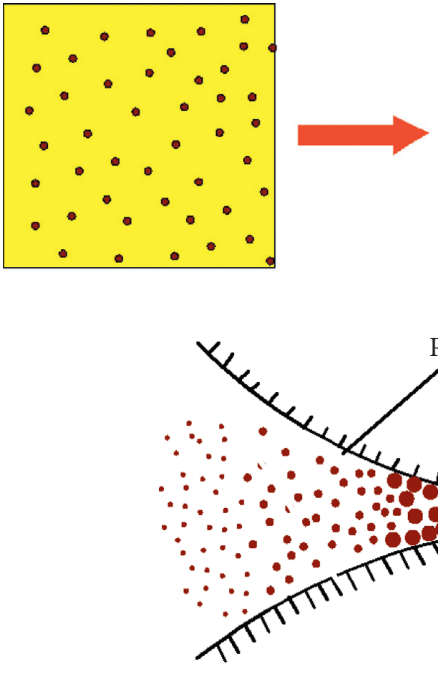
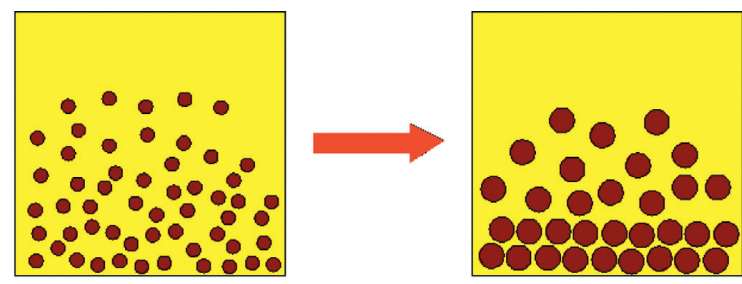

(a)

Advantages

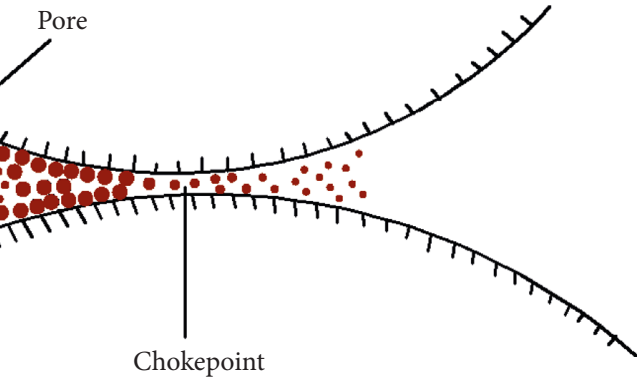

(b)

FIGURE 9: Schematic diagram of the growth and migration of polymeric microspheres through a pore chokepoint.

permeability layers, the microspheres can be adsorbed to plug the chokepoint. In a formation pore, the smaller polymeric microspheres can still migrate with the current until encountering a pore chokepoint of the same size. The experiment proved that these swollen polymeric microsphere bodies can adhere to the surface of a narrow pore opening (chokepoint) to plug it. The polymeric microspheres cracked by shearing can plug smaller chokepoints. The dual action of water-flooding pressure and shearing force fractures makes the polymeric microspheres migrate and gather toward smaller openings to plug them, resulting in a rise of injection pressure. The profile control mechanism by the polymeric microspheres is shown in Figures 8 and 9.

\section{Conclusion}

(1) The AM/AA/MMA polymeric microspheres were prepared through distillation-precipitation polymerization using acrylamide, acrylic acid, and methyl methacrylate as the monomers; $N, N$-methylene bis-dialkyl-phosphonate acrylamide as the cross-linking agent; and azoisobutyronitrile as the radical initiator.

(2) Infrared spectroscopy showed that the prepared polymeric microspheres were the copolymerization product of acrylamide, acrylic acid, and methyl methacrylate. The TGA analysis showed that the polymeric microspheres could act as oil displacement profile control agent. The polymeric microspheres were uniformly round with good sphericity. The size of the polymeric microspheres ranged from dozens to hundreds of nanometers.

(3) After the AM/AA/MMA polymeric microspheres were hydrated, the apparent viscosity of the polymeric microspheres became significantly reduced with increasing shear rate, and the fluid demonstrated a pseudoplastic behavior (a shear rate of $\left.0-100 \mathrm{~s}^{-1}\right)$. When the shear rate was $200-1000 \mathrm{~s}^{-1}$, the apparent viscosity of the polymeric microspheres did not change significantly with increasing shear rate, demonstrating a Newtonian fluid behavior. After the AM/AA/MMA polymeric microspheres were hydrated, the distribution curve of particle size showed a normal distribution. The particle size increased gradually with increasing hydration time, demonstrating the strong hydration and swelling capacity of the microspheres.

(4) The polymeric microspheres acted as a cross-linked expansive plugging agent that could largely overcome the drawbacks of traditional polymers. The microspheres demonstrated salinity and shear resistance and could penetrate pore formations. With 
excellent selective entering capacity after imbibition, it is fit for deep fluid diversion at the bottom of lowpermeability oil reservoirs. After swelling, if the polymeric microspheres were smaller than $1 / 3$ of the pore opening diameter, the polymeric microspheres entered smoothly. If the diameter was greater than $1 /$ 3 of the chokepoint diameter, they passed slowly and fractured in the case of large pressures. The polymeric microspheres deformed under the driving pressure in the immediate vicinity of a wellbore because of the large pressure difference after microsphere injection into the oil layer pore. Thereafter, the microspheres descend to the bottom of the oil layer via its pores.

(5) The core flooding test showed that polymer microsphere has both good plugging effect and good migration ability.

\section{Data Availability}

The data used to support the findings of this study are included within the article.

\section{Conflicts of Interest}

The authors declare that they have no conflicts of interest.

\section{Acknowledgments}

This work was supported by the Natural Science Foundation of Heilongjiang Province of China (Project no. E2015036), the National Science and Technology Major Projects of China for Oil and Gas (Projects nos. 2016ZX05055 and 2016ZX05012), and the Cultivation Fund of Northeast Petroleum University of China.

\section{References}

[1] C. Xiong and X. Tang, "Technologies of water shut-off and profile control: an overview," Petroleum Exploration and Development, vol. 34, no. 1, pp. 83-88, 2007.

[2] D. E. Owens, Y. Jian, J. E. Fang, B. V. Slaughter, Y.-H. Chen, and N. A. Peppas, "Thermally responsive swelling properties of polyacrylamide/poly (acrylic acid) interpenetrating polymer network nanoparticles," Macromolecules, vol. 40, no. 20, pp. 7306-7310, 2007.

[3] F. Candau, Y. S. Leong, G. Pouyet, and S. Candau, "Inverse microemulsion polymerization of acrylamide: characterization of the water-in-oil microemulsions and the final microlatexes," Journal of Colloid and Interface Science, vol. 101, no. 1, pp. 167-183, 1984.

[4] H. Jia, Q. Ren, W.-F. Pu, and J. Zhao, "Swelling mechanism investigation of microgel with double-cross-linking structures," Energy \& Fuels, vol. 28, no. 11, pp. 6735-6744, 2014.

[5] Y. Li and Z. Wen, "Analysis of effect of polymer flooding in triassic extra-low permeability reservoirs with high salinity in Northern Shaxi Oilfield," Drilling \& Production Technology, vol. 30, no. 3, pp. 36-39, 2007.

[6] Y. Li, Y. Liu, B. Bai, and G. Liu, "Research of expansion grain chemical for water shutoff and profile control," Oil Drilling \& Production Technology, vol. 21, no. 3, pp. 65-68, 1999.
[7] Z. Dong, Z. Wu, M. Lin, and M. Li, "The study of the conformation and size of the linked polymer coils," Acta Polymerica Sinica, vol. 33, no. 4, pp. 493-497, 2002.

[8] J. Wang, H. Dong, P. Li, J. Liu, and R. Wang, "In-situ polymerizing/crosslinking/gelating compositions as acid proof and high temperature resistant water plugging agent," Oilfield Chemistry, vol. 21, no. 4, pp. 313-315, 2004.

[9] X. Zhao, L. Dong, Z. Wang, Y. Bai, and M. Fu, "Application status and development tendency of deep fluid diversion," Applied Chemical Industry, vol. 42, no. 6, pp. 1121-1123, 2013.

[10] M. Lin, F. Han, M. Li, and Z. Wu, "Study on plugging performance of LPS with nucleopore film," Membrane Science and Technology, vol. 23, no. 2, pp. 11-14, 2003.

[11] C. Yao, L. Li, G. Lei, and X. Gao, "Deep profile control and flooding performance of throat-scale elastic microspheres," Journal of Southwest Petroleum University, vol. 35, no. 4, pp. 114-120, 2013.

[12] D. Li, J. Hou, F. Zhao, L. Sun, and A. Wang, "Research on profile control and water shut-off performance of precrosslinked gel particles and matching relationship between particle and pore size," Journal of Petrochemical Universities, vol. 23, no. 2, pp. 25-28, 2010.

[13] X. Cui, J. Piao, M. Li, and M. Lin, "The rheological properties of preformed particle gel dispersion system," Acta Petrolei Sinica (Petroleum Processing Section), vol. 24, no. 4, pp. 415-419, 2008.

[14] X. Li, Y. Zhang, Y. Jia, X. Liu, and T. Yang, "Application of polymer microspheres profile-controlling technology in Changqing oilfleld," Oilfield Chemistry, vol. 29, no. 4, pp. 419-422, 2012.

[15] G. Lei and J. Zheng, "Composing of pore-scale polymer microsphere and its application in improving oil recovery by profile control," Journal of China University of Petroleum (Edition of Natural Science), vol. 31, no. 1, pp. 87-90, 2007.

[16] Z. Zhang, G. Lei, Z. Liu, H. Xu, and X. Wang, "A study on profile modification by polymer microsphere injection," Xinjiang Petroleum Geology, vol. 28, no. 6, pp. 749-751, 2007.

[17] J. Li, W. Zhu, Y. Long, X. Lan, and Z. Yang, "Hydration expansion and plugging properties of nano/micron-sized polymer particles in porous media," Journal of Daqing Petroleum Institute, vol. 36, no. 3, pp. 52-57, 2012.

[18] R. Arshady, "Preparation of polymer nano- and microspheres by vinyl polymerization techniques," Journal of Microencapsulation, vol. 5, no. 2, pp. 101-114, 1988.

[19] X. Liu and J. Xu, "Analysis of contaminants in water produced from formation with polymer flooding and its possibility for recycling," Research of Environmental Sciences, vol. 14, no. 2, pp. 54-56, 2001.

[20] X. Jia, G. Lei, L. H. Yin, and R. Wang, "A study on waterswelling property of pore-scale elastic polymer microsphere," Petroleum Drilling Techniques, vol. 37, no. 6, pp. 87-90, 2009.

[21] X. Jia, G. Lei, J. Yin, C. Yao, C. Wu, and W. Li, "The relationship between pore-scale elastic microsphere and formation matching," Petroleum Drilling Techniques, vol. 39, no. 4, pp. 87-89, 2011.

[22] G. Lei, L. Li, and H. A. Nasr-El-Din, "New gel aggregates for water shut-off treatments," in Proceedings of the SPE Improved Oil Recovery Symposium, Tulsa, OK, USA, April 2010.

[23] A. Ovcharenko and M. Devadass, "Assessment on the performance of hollow glass microspheres in low density fluids for workover programs in fractured basement reservoir at the white tiger oil fields, Cuu Long Basin, Vietnamin," in Proceedings of the SPE/IADC Managed Pressure Drilling and 
Underbalanced Operations Conference and Exhibition, Kuala Lumpur, Malaysia, February 2010.

[24] X. Zhang and X. Zhou, "Experimental study on profile control and flooding with polymer elastic microsphere emulsion," Oil Drilling \& Production Technology, vol. 30, no. 5, pp. 89-92, 2008. 